Results: The frequency of heterozygote rs33996649GA genotype was higher in pSS patients than HS [OR=3.143 (1-10.234), $p=0.046]$, and also, rs33996649GA genotype was associated with high SSDAI score $(p=0.01)$. The pSS patients showed 44-fold more mRNA expression in comparison with HS $(p=0.002)$, and mRNA expression correlates with SSDAI $\left(r^{2}=0.512\right.$, $\mathrm{p}=0.006)$.

Conclusion: The rs33996649G>A genetic variant of the PTPN22 gene is associated with increased development risk of pSS in the western Mexican population. The expression mRNA correlates with disease activity in pSS.

References:

[1] Brito-Zerón, P., Baldini, C., Bootsma, H., Bowman, S. J., Jonsson, R., Mariette, X., Ramos-Casals, M. (2016). Sjögren syndrome. Nature Reviews Disease Primers, 2(July), 1-20. https://doi.org/10.1038/nrdp.2016.47

[2] Stanford, S. M., \& Bottini, N. (2014). PTPN22: The archetypal non-HLA autoimmunity gene.Nature Reviews Rheumatology, 10(10), 602-611.https://doi. org/10.1038/nrrheum.2014.109

[3] Chen, Z., Zhang, H., Xia, B., Wang, P., Jiang, T., Song, M., \& Wu, J. (2013). Association of PTPN22 gene (rs2488457) polymorphism with ulcerative colitis and high levels of PTPN22 mRNA in ulcerative colitis. International Journal of Colorectal Disease, 28(10), 1351-1358. https://doi.org/10.1007/ s00384-013-1671-3

[4] Machado-Contreras, J. R., Muñoz-Valle, J. F., Cruz, A., Salazar-Camarena, D. C., Marín- Rosales, M., \& Palafox-Sánchez, C. A. (2016b). Distribution of PTPN22 polymorphismsin SLE from western Mexico: correlation with mRNA expression and disease activity. Clinical and Experimental Medicine, 16(3), 399-406. https://doi.org/10.1007/s10238-015-0359-0

Disclosure of Interests: None declared

DOI: 10.1136/annrheumdis-2020-eular.2173

\begin{tabular}{l|l}
\hline AB0017 & CONSTANT GENETIC MARKER IL1B T-31C IS \\
ASSOCIATED WITH ANAMNESIS OF BIOLOGICAL \\
DRUGS TREATMENT IN RHEUMATOID ARTHRITIS
\end{tabular}

V. Omelchenko ${ }^{1}$, E. Letyagina ${ }^{1}$, Y. Kurochkina ${ }^{1}$, A. Akimova ${ }^{1}$, A. Shevchenko ${ }^{1}$ M. Korolev ${ }^{1}{ }^{1}$ RICEL - Branch of IC\&G SB RAS, Novosibirsk, Russian Federation

Background: Rheumatoid arthritis (RA) is chronic progressive joint disease with erosions formation. Timely and effectiveness treatment is important due to quickly structural damage and progressive losing of active motion. Synthetic DMARDs didn't have a sufficient effect. Using biological drugs seemed like a panacea, but according to investigations at least 30-40\% RA-patients lost treatment efficiency. Biological drugs act through immune cascade, that's why mutation in regulatory region of cytokine genes may partly determine treatment failure.

Objectives: The objective of our study was to analyze the frequency of IL1T-31C single nucleotide polymorphism in patient with rheumatoid arthritis and its association with biological drugs prescribing.

Methods: One hundred two Caucasian RA-patients (age - 56 yrs [45; 61]; DAS28 4.7 [3.8; 5.9]) were enrolled in our study. All of them had American College of Rheumatology (ACR)-defined RA (1987 classification criteria) and gave written inform consent. Single nucleotide polymorphisms IL1B T-31C (rs1143627), IL4 C-590T (rs2243250), IL10 C-592A (rs1800872), IL10 A-1082G (rs1800896) were determined by restriction fragment length polymorphism. Descriptive statistics, Chi-squared test were used for data analysis. Results are presented as median and 25th/75th percentiles (Me [25th percentile; 75th percentile]).

Results: The most of SNPs analyzed had corresponded to the Hardy Weinberg equilibrium (HWE). The only exception was IL1B T-31C - the frequencies were differed statistically significant from HWE $(p=0,03)$. Forty seven $(46.1 \%)$ patients were treatment with biological drugs. Homozygotes IL1b -31CC were founded more frequently beside patients with biological treatment compare with other group (13 from $47(27,7 \%)$ vs. 6 from $52(11.5 \%), p=0,042)$. Other SNPs didn't demonstrate any associations.

Conclusion: Single nucleotide polymorphism IL1B T-31C (rs1143627) may be used for prognosis of basic anti-inflammatory therapy inefficiency and the needing for prescribing biological therapy.

Disclosure of Interests: None declared

DOI: 10.1136/annrheumdis-2020-eular.2404

\section{AB0018 $\quad$ TNFA RS1800629 POLYMORPHISM: WHAT ABOUT ITS ASSOCIATION WITH CLINICAL MANIFESTATIONS AND ANTI-TNFA THERAPY? DATA FROM A SERIES OF ITALIAN PATIENTS WITH BEHÇET SYNDROME}

M. C. Padula ${ }^{1,2}$, P. Leccese ${ }^{1}$, N. Lascaro ${ }^{1}$, G. G. Sorrento ${ }^{2}$, R. P. Radice ${ }^{2}$, A. R. Limongi ${ }^{2}$, T. Carbone ${ }^{1}$, A. Padula ${ }^{1}$, G. Martelli ${ }^{2}$, S. D'angelo ${ }^{1} .{ }^{1}$ Rheumatology Institute of Lucania (IReL), San Carlo Hospital of Potenza, Potenza, Italy;

${ }^{2}$ University of Basilicata, Potenza, Italy
Background: Tumor Necrosis Factor-alpha (TNF-a) is a pleiotropic cytokine with a critical role in the pathogenesis of Behçet syndrome (BS). Anti-TNFa therapy is useful for patients with refractory, severe BS, in particular for ocular, central nervous system, and gastrointestinal manifestations. However, although biological treatment with anti-TNF-a agents are effective in BS, not all patients are definite responders. Non-responders patterns could be due to: alternative non-TNFa related pathway of inflammation; anti-drug antibodies presence or development; polymorphic alleles of $T N F a$ gene. TNFa rs1800629 (-308G $>A)$ is a drug-response single nucleotide polymorphism (SNP) located within the gene promoter. Poor and conflicting data are currently available about the association of this polymorphism and clinica manifestations of BS, as well as about the responsivness to the TNFa blockers in BS patients [1-3].

Objectives: Aims of this study were to investigate in a cohort of Italian patients with BS the frequency of rs 1800629 genotypes and its association with clinical features and anti-TNFa therapy response.

Methods: Consecutive patients with BS were recruited. Patients demographic and clinical data were collected by medical records and analyzed. Home-made specific primer pairs were used for rs 1800629 coverage. gDNA was isolated and amplified using PCR. Good-quality amplicons were sequenced (Sanger method) In silico analysis was downstream performed using specific software for querysubject similarity analysis.

Results: 130 BS patients (64M:66F; mean age: $45.8 \pm 12.3$ years) were included in the study. Patients predominant lesions were oral aphtosis $(100 \%)$, eye involvement $(86.2 \%)$, skin lesions $(72.3 \%)$ and genital ulcers (57.7\%). TNFa rs 1800629 wild-type GG genotype was found in 106/130 BS patients $(81.5 \%)$; the heterozygous genotype (GA) was identified in $24 / 130$ patients $(18.5 \%)$. No statistically significant differences were found in genotypes frequencies when the patients were stratified for presence and absence of each clinical manifestation $(p>0.05)$, while statistical significant differences were found when the patients were compared for therapy (antiTNFa drugs) response. In detail, $73 / 130$ patients $(56.2 \%)$ were treated with anti-TNFa agents. We found $16 / 73(21.9 \%)$ non-responders patients (NRP). In NRP group, we identified $9 / 16$ patients (56.3\%) with GG genotype and $7 / 16$ $(43.7 \%)$ with GA genotype, while $8 / 57(14.0 \%)$ responder patients showed GA genotype and $49 / 57$ responder patients (86.0\%) showed GG genotype $(p=0.0093$; OR: 0.21, Cl: 0.06-0.729).

Conclusion: Here we described a low frequency of TNFa rs1800629 SNPcontaining allele and the lack of association between SNP and BS clinical hallmark, as previously reported in literature [1-4]. We also found higher percentage of GG genotype in case of therapy response than GA genotype. The SNP is a promoter polymorphism that could affect the auto-inflammatory response and the therapy responsivness, as suggested by our preliminary data of pharmacogenomics. Analyses of a larger cohort of patients are need to confirm the study findings and to explain the SNP role as outcome predictor.

\section{References:}

[1] Touma Z et al. (2010). Arch Med Res 41(2):142-6;

[2] Vallet $\mathrm{H}$ et al. (2015). J Autoimmun 62:67-74

[3] Zhang M et al. (2013). Mol Vis 19:1913-24.

[4] Ateş A et al. (2006). Rheumatol Int 26(4):348-53.

Disclosure of Interests: Maria Carmela Padula: None declared, Pietro Leccese: None declared, Nancy Lascaro: None declared, Giusi Gaia Sorrento: None declared, Rosa Paola Radice: None declared, Antonina Rita Limongi: None declared, Teresa Carbone: None declared, Angela Padula: None declared, Giuseppe Martelli: None declared, Salvatore D’Angelo Consultant of: AbbVie, Biogen, BMS, Celgene, Eli Lilly, MSD, Novartis, and UCB, Speakers bureau: AbbVie, BMS, Celgene, Eli Lilly, Novartis, Pfizer and Sanofi

DOI: 10.1136/annrheumdis-2020-eular.2295

\section{AB0019 \\ GENETIC POLYMORPHISM OF THE INFLAMMATORY MARKER SAA1 RS12218 (-13 T/C) IS ASSOCIATED WITH AN APTITUDE TO CLINICAL PHENOTYPES OF JUVENILE IDIOPATHIC ARTHRITIS}

E. Fedorov ${ }^{1}$, S. Salugina ${ }^{2}$, M. Krylov ${ }^{3}$, I. Guseva ${ }^{3}$, E. Samarkina ${ }^{3}{ }^{1}$ V.A. Nasonova Research Institute of Rheumatology, Pediatric, Moscow, Russian Federation; ${ }^{2}$ V.A. Nasonova Research Institute of Rheumatology, Pediatric, Moscow, Russian Federation; ${ }^{3}$ V.A. Nasonova Research Institute of Rheumatology, Immunology and Molecular Biology of Rheumatic Diseases, Moscow, Russian Federation

Background: Juvenile idiopathic arthritis (JIA) is one of the most widely-spread immuno-inflammatory diseases of an unknown etiology, the leading manifestation of which is chronic joint inflammation, occuring in children under the age of 16. The disease is a complex of chronic arthropathies with various phenotypic manifestations. 
Objectives: To verify the hypothesis about the role of SAA1 rs12218 T / C gene polymorphism in the aptitude to various clinical JIA phenotypes.

Methods: The study included 132 children, of whom 66 were diagnosed with JIA and 66 were healthy unrelated volunteers (the college students) as a control group, comparable by gender and age. The group of patients with JIA consisted of 44 girls and 22 boys, with an average age of $11.7 \pm 4.2$ years and an average disease duration of $4.8 \pm 3.8$ years. The diagnosis and classification of JIA was established according to ILAR-2004 criteria. The JIA group included 30 (45\%) patients with oligoarthritis (oJIA), of which 20 patients $(67 \%)$ were positive for the HLA-B27 antigen (JIA-B27 +) and 10 (33\%) patients with anterior uveitis (uJIA); $20(30 \%)$ patients were assigned to the group with the polyarticular variant (pJIA), while all of them were seronegative for the rheumatoid factor; 16 (24\%) patients were diagnosed with JIA with a systemic onset (SJIA). The frequencies of the T / C polymorphism of the SAA1 gene were assessed using an allele-specific polymerase chain reaction in a real time mode (RT-PCR).

Results: In the group of patients diagnosed with oJIA and (JIA-B27 +), the frequency of the $\mathrm{C}$ allele was significantly higher compared to the control $(53.3 \%$ and $57.5 \%$ versus $37.1 \%, p=0.035$ and 0.022 , respectively). No significant differences were detected in the frequencies of the mutant $C$ allele between SJIA and pJIA and the control group. The logistics analysis of the frequency distribution of the alleles of the SAA1 gene demonstrated an increased risk of the $\mathrm{C}$ allele in formation of an aptitude to the oJIA variant (OR 1.94, 95\% Cl 1.00-3.76, $\mathrm{p}=$ $0.035)$. In the oJIA-B27 (+) group, the risk of an aptitude was also increased compared to the control (OR 2.29, 95\% Cl 1.05-5.04, $\mathrm{p}=0.022$ ).

Conclusion: The data obtained confirm for the first time the involvement of the rs12218 polymorphism of the SAA1 gene in an aptitude to the oligoarthritis JIA clinical phenotype. The presented results require further replication researches using an enlarged number of patients from different population groups.

Disclosure of Interests: None declared

DOI: 10.1136/annrheumdis-2020-eular.4119

\section{AB0020 \\ CORRELATION BETWEEN SERUM AND SYNOVIAL CONCENTRATION OF IL-17A AND MIRNA EXPRESSION IN RHEUMATOID ARTHRITIS PATIENTS}

R. Shumnalieva ${ }^{1}$, D. Kachakova ${ }^{2}$, T. Velikova ${ }^{3}$, R. Kaneva ${ }^{2}$, Z. Kolarov ${ }^{1}$, S. Monov ${ }^{1}{ }^{1}$ Medical University - Sofia, Bulgaria, Department of Internal Medicine, Clinic of Rheumatology, Sofia, Bulgaria; ${ }^{2}$ Medical University - Sofia, Department of Medical Chemistry and Biochemistry, Molecular Medicine Center, Sofia, Bulgaria; ${ }^{3}$ Clinical Immunology, University Hospital Lozenetz, Sofia, Bulgaria

Background: Interleukin 17 (IL-17) is a proinflammatory cytokine, which overproduction promotes the autoimmune reaction in rheumatoid arthritis (RA). Posttranscriptional regulation of IL-17 by specific microRNAs (miRNAs) is of great interest in the recent years. 146a was associated with IL-17 expression in IL-17 producing T-cells in the synovium when miR-155 enhanced Treg and Th17 cells differentiation and IL-17A production by directly targeting the suppressor of cytokine signaling (SOCS) 1 [1, 2]. It has been shown that IL-17 production in lymphocytes or its function could be regulated by miR-223 by targeting Roquin ubiquitin ligase or its receptors [3]

Objectives: To examine a possible correlation between systemic and local concentrations of IL-17A and systemic and local miR-146a, miR-155 and miR-223 expression in RA patients.

Methods: Expression levels of three miRNAs were determined in matched peripheral blood (PB) and synovial fluid (SF) samples of RA patients by relative quantitation method $2^{-\Delta \Delta C t}$. As reference control for normalization RNU6B gene was used. Concentrations of IL-17A were compared between matched serum and SF samples from 20 RA patients by Human IL-17A ELISA kit (Gene probe, Diaclone, France). Healthy donors were used as controls.

Results: miR-146a, miR-155 and miR-223 showed overexpression in RA SF when compared to HCs SF (in $70.83 \%, p=0.007$; in $79.17 \%, p=1.63 \times 10^{-4}$ and in $79.17 \%, p=1.64 \times 10^{-3}$, respectively). The ROC curve analysis showed diagnostic accuracy for miR-146a in SF with $A \cup C=0.769, p=0.006, A \cup C$ for SF miR-155 was $0.858, p=2.3 \times 10^{-4}$ and AUC for SF miR-223 was $0.841 p=4.6 \times 10^{-4}$. SF levels of miR-146a and miR-155 were overexpressed in $52.17 \%$ and in $76.09 \%$ of the RA patients compared to its systemic levels. SF miR-223 was underexpressed in $58.7 \%$ of the patients compared to its systemic levels. Levels of IL-17A were higher in RA SF compared to serum (8.645 pg/ml versus $0.315 \mathrm{pg} / \mathrm{ml}, \mathrm{p}=0.012$ ). ROC curve analysis for SF IL-17A showed area under the curve $(A \cup C)=0.885$, $\mathrm{p}<0.000$.

Conclusion: The difference between the systemic and local concentration of $\mathrm{IL}-17 \mathrm{~A}$ and miRNAs expression shows that the inflammatory disease process leads to their altered expression with a possible role of these molecules in the disease pathogenesis. The higher local levels of miR-155, miR-146 and IL-17A confirm the data about the possible role of these miRNAs in regulating IL-17A production. The opposite changes of IL-17A and miR-223 systemic and local levels confirm the data about the possible role of miR-223 in regulating IL-17 function. Further analysis with larger sets is needed to confirm these results. References:

[1] Niimoto T, Nakasa T, Ishikawa M, Okuhara A, Izumi B, Deie M, et al. MicroRNA-146a expresses in interleukin-17 producing T cells in rheumatoid arthritis patients. BMC Musculoskelet Disord. 2010; 11:209.

[2] Yao R, Ma YL, Liang W, Li HH, Ma ZJ, Yu X. et al. MicroRNA-155 modulates Treg and Th17 cells differentiation and Th17 cell function by targeting SOCS1. PLoS ONE 2012; 7(10):e46082.

[3] Schaefer J, Nakra N, Montufar-Solis D, Vigneswaran N, Klein J. Role for miR223 and Roquin in IL-10 mediated regulation of IL-17. J Immunol. 2013; 190 (1 Supplement) 171.9.

Acknowledgments: The study was supported by Grant 14-D/2012, Grant 60/2013 and Grant 61/2015 from Medical University-Sofia, Bulgaria

Disclosure of Interests: None declared

DOI: 10.1136/annrheumdis-2020-eular.6226

\section{$\mathrm{AB} 0021$ \\ VARIABILITY OF THE RS333 IN LUPUS ERYTHEMATOSUS PATIENTS FROM THE POLISH POPULATION}

K. Skonieczna ${ }^{1}$, D. Mlicka ${ }^{1}$, A. Woźniacka ${ }^{2}$, R. Czajkowski ${ }^{3}$, E. Robak ${ }^{2}$, M. Gawrych ${ }^{3}$, A. Duleba 1 , T. Grzybowski ${ }^{1}{ }^{1}$ Collegium Medicum, Nicolaus Copernicus University, Department of Forensic Medicine, Bydgoszcz, Poland; ${ }^{2}$ Medical University of Łódź, Department of Dermatology and Venereology, Łódź, Poland; ${ }^{3}$ Collegium Medicum, Nicolaus Copernicus University, Department of Dermatology and Venereology, Bydgoszcz, Poland

Background: There are several subtypes of Lupus Erythematosus (LE), which may be limited to the skin (eg. Discoid Lupus Erythematosus, DLE) or involve multiple organ dysfunctions (Systemic Lupus Erythematosus, SLE). LE is an autoimmune disease that is influenced by genetic and environmental factors. Despite some genetic changes between DLE and SLE were previously shown, the complete genetic background of DLE is still unresolved [1]. Functional C-C chemokine receptor 5 (CCR5) receptor can be associated with the inflammation in LE patients. Importantly, the 32 base pairs (bp) deletion in CCR5 gene (rs333) leads to a nonfunctional receptor. Previous studies have shown that this mutation may have a protective effect on the development and progression of SLE [2, 3]. Thus it was important to investigate whether 32 bp deletion in rs333 is also associated with DLE development.

Objectives: The aim of this study was to investigate the variability of the CCR5 gene, within a polymorphic locus rs333 in SLE and DLE patients from the Polish population.

Methods: 120 LE patients (77 SLE patients and 43 DLE patients) and 100 healthy persons were recruited to the study from the Polish population. DNA was isolated from blood or buccal swabs. rs333 was genotyped by using polymerase chain reaction (PCR). Statistical significance of the differences between patient and control groups in both allele and genotype frequencies were calculated using Chi-Squared test with Yates correction or two tailed Fisher's exact test.

Results: Deletion allele of the rs333 was significantly less frequent among DLE patients than healthy persons $(p=0.0171)$. Also the heterozygotes occu significantly less frequent within DLE patients group than in healthy individuals $(p=0.0375)$. Moreover, homozygotes without deletion in rs333 were found significantly more frequent in persons diagnosed with DLE than in healthy volunteers $(p=0.0214)$. In contrast, the differences in allele or genotype frequencies between SLE patients and healthy controls were not statistically significant ( $p>0.05)$. Moreover, the rs333 variability was not associated with clinical symptoms of LE patients $(p>0.05)$.

Conclusion: Summarizing, the results obtained in this study suggest that the $32 \mathrm{bp}$ deletion within rs333 could be a protective factor, that reduce the risk for DLE but not SLE development in the Polish population. However, due to the low statistical power of the obtained results, further studies on larger groups of patients and controls are needed to acquire more reliable data.

\section{References:}

[1] Skonieczna K, Czajkowski R, Kaszewski S, Gawrych M, Jakubowska A, Grzybowski T. (2017) Genetic similarities and differences between discoid and systemic lupus erythematosus patients within the Polish population. Postepy Dermatol Alergol. 34: 228-232.

[2] Schauren JS, Marasca JA, Velt TD, Monticielo OA, Xavier RM, Brenol JCT et al (2013) CCR5 delta32 in systemic lupus erythematosus: implications for disease susceptibility and outcome in a Brazilian population. Lupus 22:802-809.

[3] Martens, H.A., Kallenberg, C.G. \& Bijl, M. (2009) Role of CCR5 Delta32 bp deletion in RA and SLE. Autoimmunity, 42, 260. 